\title{
Assessing the Possibility of Identifying Precancerous Cervical Lesions using Aceto- White Temporal Patterns
}

\author{
Héctor-Gabriel Acosta-Mesa ${ }^{1}$, Nicandro Cruz-Ramírez" ${ }^{1}$, \\ Karina Gutiérrez-Fragoso ${ }^{1}$, \\ Rocío-Erandi Barrientos-Martínez ${ }^{1}$ and Rodolfo Hernández-Jiménez ${ }^{2}$ \\ ${ }^{1}$ School of Physics and Artificial Intelligence, Department of Artificial Intelligence, \\ Universidad Veracruzana, Sebastián Camacho \# 5, 91000, Xalapa, \\ ${ }^{2}$ Obstetrician and Gynaecologist, Diego Leño \# 22, C.P. 91000, Xalapa, \\ Ver. México.
}

\section{Introduction}

In 2005, according to projections by the World Health Organization (WHO, 2007), there are more than 500000 new cases of cervical cancer worldwide, $90 \%$ of these occur in developing countries. It is estimated that over 1000000 women currently suffer from cervical cancer in the world. However, most of them has not been diagnosed or do not have access to necessary treatments. That same year, about 260000 women died of this disease and about $95 \%$ of these deaths occurred in developing countries. Cervical cancer has remained one of the most serious threats to women's lives (WHO, 2007).

After Pap smear test, colposcopy is the most used technique to diagnose this disease because of its a higher sensitivity and specificity. One of the most important colposcopic findings is the level of white color intensity that the cervical tissue reaches after acetic acid application. The major problem with this technique is the intrinsic subjectivity of the test i.e. the amount and speed of color change perceived could be different for various observers; this fact may produce high variability on the diagnosis made by these experts. So the mechanisms to quantify near to absolute measurements of color and speed changes are needed to improve the test. It is difficult to find in the literature a general accepted score for the sensitivity and specificity reached by an expert colposcopist, but in average this test has high sensitivity (98\%) but low specificity (48\%) (Vlastos, 2002).

Some researchers have suggested the use of the temporal patterns intrinsic to the color changes (Balas, 2001; Stefanaki et al., 2001; Pogue Brian et al., 2001). Although some efforts have been made to characterize precancerous cervical lesion using aceto-white temporal patterns, to the best of our knowledge, there is not a complete understanding of how to use them to segment colposcopic images, and there is not an established methodology to automatically analyze colposcopic images using aceto-white temporal patterns for classification of cervical tissue.

In the present work, we compare the shape of the temporal patterns to establish relationships among similar shapes, and the correlation of those patterns with certain types

Source: Decision Support Systems, Advances in, Book edited by: Ger Devlin,

ISBN 978-953-307-069-8, pp. 342, March 2010, INTECH, Croatia, downloaded from SCIYO.COM 
of tissue. Our analysis not only shows results of those small areas from where the biopsies were acquired, but also considers the segmentation of the complete image, which is more realistic. This is particularly important since in a real computer aided system, the analysis of the complete image is needed, considering noisy data and outliers. We use a discretized version of the raw temporal pattern to develop a supervised Machine Learning (ML) processes, using one of the most effective probabilistic classifier Naïve Bayes (NB) (Friedman, 1997; Han, 2001; Duda, 2001).

The paper is structured in four sections: in the first section, the technical issues of subject preparation and data acquisition are explained, in the second part, data analysis methodology is explained, including image acquisition and preprocessing, time series extraction and discretization and machine learning implementation. In the third section results of the application of the methodology on real data of 38 patients are shown. Finally, in the fourth section some conclusions and future work are commented.

\section{Materials and methods}

\subsection{Subject preparation}

Thirty eight women with abnormal Papanicolaou, ages ranging from 22 to 35 years participated in the experiment. All of them gave informed written consent. Before colposcopy, the cervical mucus was cleaned using a cotton-wool swabs. The colposcopic tests were made spreading three milliliters of acetic acid $(3 \%)$ over the cervix using a needle for fast application. A cotton-wool was put in the low part of the cervix to absorb the remaining acetic acid that drops after the application. A leg-holder structure was used to make the patient feels comfortable and to reduce movements. After colposcopy, a biopsy was taken for histological analysis and PCR test (Anderson, 1993).

\subsection{Data acquisition}

Images were acquired using a colposcope $\mathrm{dfv}$ Vasconsellos model CP-M7 with magnification $16 \mathrm{X}$ without any optical filter. The viewing distance was $20 \mathrm{~cm}$. and the field of view covers approximately a circle of $13 \mathrm{~mm}$ ratio. Images were acquired using a color camera Sony SSC-DC50A and a frame grabber Matrox Meteor-II/Standard driven by a HP workstation XW6000 running Matlab 7.0 image acquisition toolbox. During the first ten seconds of the image acquisition, 10 images $(352 \times 240)$ were taken as base line reference (1 frame/second), then after acetic acid application, three hundred images were taken in 5 minutes using the same sampling frequency. Control images taken at the beginning of each trial have a double purpose, the first one is to have a base reference to assess the signal percentage of change and the second one is to estimate the amount of signal noise. Each image was saved independently as a BMP file. Although we developed analysis over color images (results not shown in this article), in this publication we present results obtained processing the images in gray scale because results were similar.

\section{Data analysis}

The colposcopic image sequence can be represented as a sequence of $t 2 \mathrm{D}$ images $\mathrm{I}_{\mathrm{t}}(\mathrm{x}, \mathrm{y})$ with acquisition time $t$ with $t<t+1$. The color variation over time of each pixel in the image provides a time series. The resulting image sequence can be viewed as a $3 \mathrm{D}$ image block $\mathrm{I}(\mathrm{x}, \mathrm{y}, \mathrm{t})$ defined on the spatio-temporal domain. The methodology proposed in this paper to 
analyze the colposcopic sequences involves 3 main processes: image registration, time series extraction and supervised learning. Those processes are explained in detail in the following sections.

\subsection{Data acquisition}

The acquisition process of colposcopic images spans over 5 minutes. Even though the patient is fixed some, small random movements are unavoidable. They have often local character (patient's breathing, movements due to the muscle tonus, etc.). To be able to analyze the sequence of the images, the structures in the images should be brought into the same position by removing the differences due to the patient movements - the colposcopic images have to be registered. This step is essential in this application; the goal of the 2D image spatial alignment is to enable comparison between corresponding anatomical positions. There are various registration methods, a good overview can be found in (Brown, 1992). The appropriate method has to be chosen with respect to the expected geometric differences and the type of processed data.

Our previous experimental assessment suggests that the main source of the misalignments in colposcopic sequences can be modeled by simple translation (Acosta-Mesa et al., 2005). The method can transform the whole data using the same parameters, or can be local, depending on the local variations. It can be based directly on the image intensity values (area-based methods) or can be carried out using some features computed from the images (feature-based methods). Because colposcopic images do not contain many distinctive details, an area-based method was chosen. The classical representative of the area-based methods is the normalized cross-correlation (CC), this method exploits directly image intensities, this measure of similarity is computed for window pairs from the input and reference images and its maximum is searched (Zitova, 2003).

The input and the reference images are updated continuously starting with the first and second images of the sequence respectively, then the input and the reference images are redefined by the second and the third images and so on. The starting points to initialize the search are updated by the last position in which the pattern window was found. This registration strategy allows not only to contend with the fact that the searched pattern changes over time, but also, to reduce the spatial space over which to develop the search. Because cervical lesions are spread over the tissue as regions (forming areas with homogeneous tissue), a high spatial resolution is not needed. Then after registration the spatial resolution was reduced at $50 \%$ of the original size.

\subsection{Time series extraction}

The colposcopic set of images can be thought of as a spatiotemporal data matrix. Let ImageCube $(m, n, t)$ represent a stack of $t$ images of size $(m, n)$. Thus there are $(m \times n)$ pixels, each of which is a time series of length $t$. Let $\mathrm{p}(\mathrm{i}, \mathrm{j})$ represent the color of the pixel $(\mathrm{i}, \mathrm{j}), \mathrm{i}=1, \ldots$, $m, j=1, \ldots, n$. The intensity value of each pixel over time is used to construct a time series, which we call, the Aceto-white response function (Awrf). Figure 1 shows an example of aceto-white response function. In order increase the signal to noise ratio, the signal was smoothed using a polynomial approximation. As a way of standardization to permit comparison among different subjects, the values of each temporal pattern are divided by the mean value of its basal period (Acosta-Mesa et al., 2007). The resultant amplitude of the Awrf is then measured as a percentage of change with respect to the base line. 

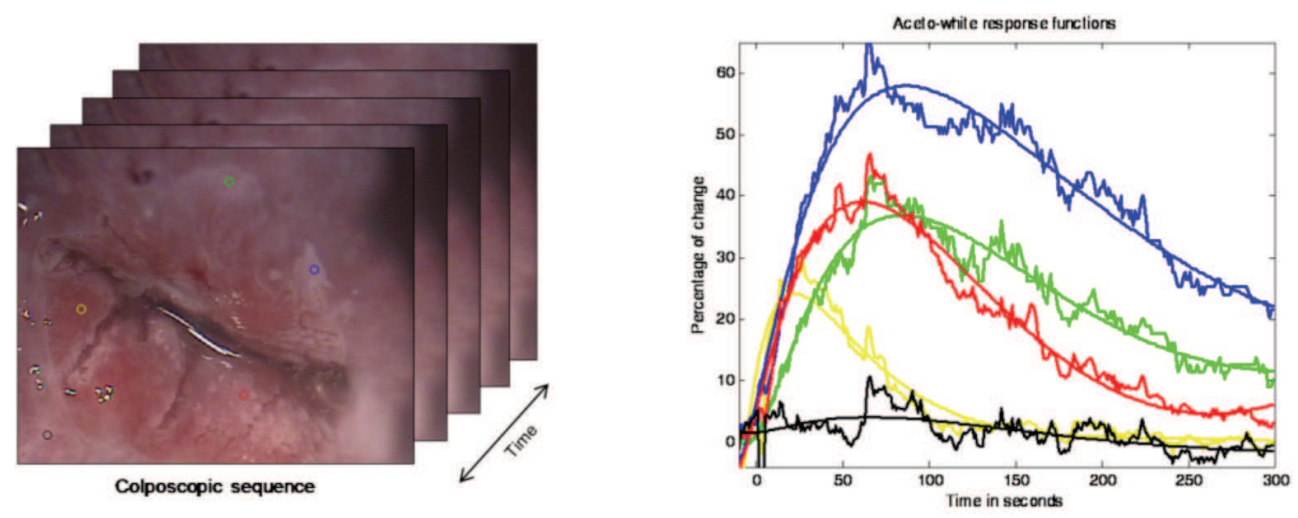

Fig. 1. Aceto-white response functions. At the right, temporal patterns observed on the pixels denoted by dots on the left image. As can be appreciated, different kinds of tissue produce Awrf with different percentage of change, speed of increase and decay. Next to each raw Awrf its polynomial fitted model is shown.

Time series databases are often extremely large. Due to the size of many databases of time series, methods have been developed to reduce the dimensionality. These methods produce approximations of time series through discretization schemes. The discretization process is focused on mapping variables with continuous values into discrete values. This mechanism has been widely used to compress data and facilitate their computational treatment. It is necessary to specify a set of parameters for the processing of data. The reduction of dimensionality in the $\mathrm{x}$-axis is obtained by dividing the total length of the time series into fragments of a certain size (word size). It is also necessary to establish a number of intervals in the $y$-axis to compress the values of the time series (size of the alphabet) (Keogh \& Pazzani, 2000). In this case, the parameters of word size and alphabet were selected experimentally. The time series were discretized with two criteria:

a. Piecewise Linear Approximation (PLA). The method involves setting the parameters of word size and alphabet for discretized time series and calculates the average of the signal in each segment (Allgower, 1988). In this case we used alphabet and word size of 10.

b. Piecewise Slope Approximation (PSA). This method is similar the one above, sets the parameters of word size and alphabet, but is based on calculating the values of slopes for each segment. In this case 7 possible values were used for the slopes describing the temporal pattern: 3 negative values, 0 representing no change and 3 positive values. Figure 2 illustrates an example of a temporal pattern that was discretized by these methods.

\subsection{Supervised learning}

Supervised learning is a machine learning area for constructing models from a set of observations presented as examples. The goal of these methods is to predict the class label of an unseen observation given the knowledge (model) extracted from the training data. Training data can be seen as a data base containing observations and their corresponding class label. When the class label value is categorical, supervised learning is called neighbors, decision trees, neural networks, naïve bayes, etc. The selection of the method 


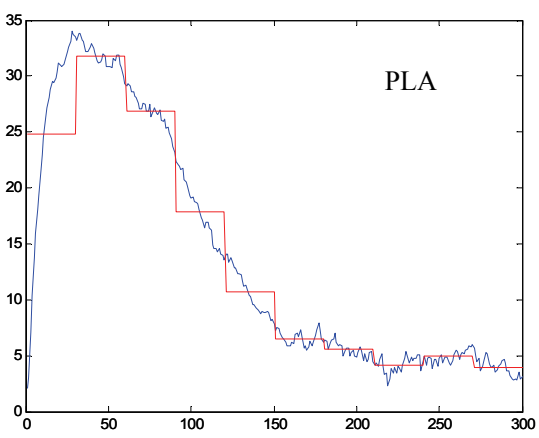

$\begin{array}{llllllllll}2 & 3 & 2 & 1 & 1 & 0 & 0 & 0 & 0 & 0\end{array}$

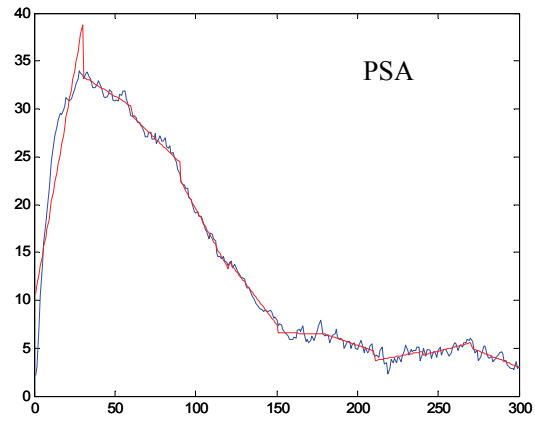

$\begin{array}{llllllllll}2 & 0 & 0 & -1 & 0 & 0 & 0 & 0 & 0 & 0\end{array}$

Fig. 2. Example of a temporal pattern that was discretized. On the left the PLA method that calculates the average of each segment is shown and on the right the PSA method, based on the values of the slopes that describe the time series is shown.

depends on the kind of data and prior hypothesis, and most of the time has to be selected empirically (Mitchell, 1997; Pang-Ning et al., 2006; Hastie, 2001).

A colposcopic data set can be seen as a time series database, the classification (segmentation) task consists of finding similar temporal patterns with regular shapes belonging to the same type of tissue (class label). In this work we are interested in investigating the relationship between regular shapes and the class tissue. We propose to use the PLA representation of raw Awrf and the Naïve Bayes classifier. As will be explained this method is a direct way to explore the relationship between the shapes of temporal patterns and their corresponding type of tissue.

\subsubsection{Naïve Bayes algorithm}

The Naïve Bayes classifier (NB) is one of the most effective classifiers (Friedman, 1997; Duda, 2001) and against which state of the art classifiers have to be compared. Its main appeals are its simplicity and accuracy: although its structure is always fixed, i.e. the class variable has an arc pointing to every attribute (assumes linear independence among the attributes), it has been shown that this classifier has high classification accuracy and optimal Bayes' error (Han, 2001; Duda, 2001). In simple terms, the NB learns, from a training data sample, the conditional probability of each attribute given the class. Then, once a new case arrives, the NB uses Bayes' rule to compute the conditional probability of the class given the set of attributes selecting the value of the class with the highest posterior probability. This rule is shown in equation 1.

Bayes' Theorem

$$
p\left(c_{j} \mid d\right)=\frac{p\left(d \mid c_{j}\right) p\left(c_{j}\right)}{p(d)}
$$

Where:

$p\left(c_{j} \mid d\right)=$ probability of class $c_{j}$ given instance $d$ $p\left(d \mid c_{j}\right)=$ probability of instance $d$ given class $c_{j}$ 
$p\left(c_{j}\right)=$ probability of occurrence of class $c_{j}$

$p(d)=$ probability of occurrence of instance $d$

\subsubsection{Classification accuracy}

Performance of classification algorithms is evaluated using different metrics, the most common are accuracy, sensibility and specificity. All of them use a confusion matrix (see table 1) to evaluate the percentage of observation assigned to the correct class label during classification. In a training data set with binary class labels we have:

\begin{tabular}{|c|c|c|}
\hline Real/ Predicted & Positive & Negative \\
\hline Positive & True Positive & False Negative \\
\hline Negative & False Positive & True Negative \\
\hline
\end{tabular}

Table 1. Confusion matrix.

Where the accuracy on the classification process is computed as the rate of true positives plus true negatives against the total number of observations; the sensibility is the rate of true positive observations against the sum of true positive plus false negatives and the specificity is the rate of true negative observations against the sum of true negative plus false positives. All those metrics to estimate classification performance are computed repeatedly partitioning the data base in two sets, the first set is used to train the algorithm and the second one is used to validate the performance of the classifier, this process is called crossvalidation (Mitchell, 1997). In the application described in the present paper, the training set is conformed by the observation of all patients minus one, the validation set is conformed by the observation of the patient that was excluded to the training set and so for all the cases. This particular case of cross validation is called leave-one-out cross- validation.

\section{Methodology and results}

Image registration was carried out using the cross-correlation technique explained above. The search window was defined selecting a region feature over which some anatomical features show high contrast boundaries, e.g. the cervical hole. In those cases in which the cervical hole was not a good reference a stain landmark was introduced using lugol solution (Anderson, 1993). Once the dataset was registered, we asked the expert colposcopist define points (pixels) on the colposcopic sequence of each patient to assign a class label on representative regions. To help the expert to do the class labeling, we develop a knowledge acquisition graphical interface where the expert can see the entire image of the colposcopic sequence. Also he was able to select the images at different times to look the aceto-white changes (see figure 3).

Based on the results reported by histology, the expert selected a pixel of the image in order to assign one of the following class labels: normal tissue, immature metaplasia, mature metaplasia, ectopy, low grade lesion, high grade lesion.

In the database each observation represents one temporal pattern. The number of observations is bigger than the number of patients because multiple pixels were selected from one region and in some patients more than one type of tissue were sampled. The cases were divided into two main classes or types of tissue: negative (normal) and positive (abnormal). The class negative includes: normal tissue $(11,851)$ (patients and observations, 


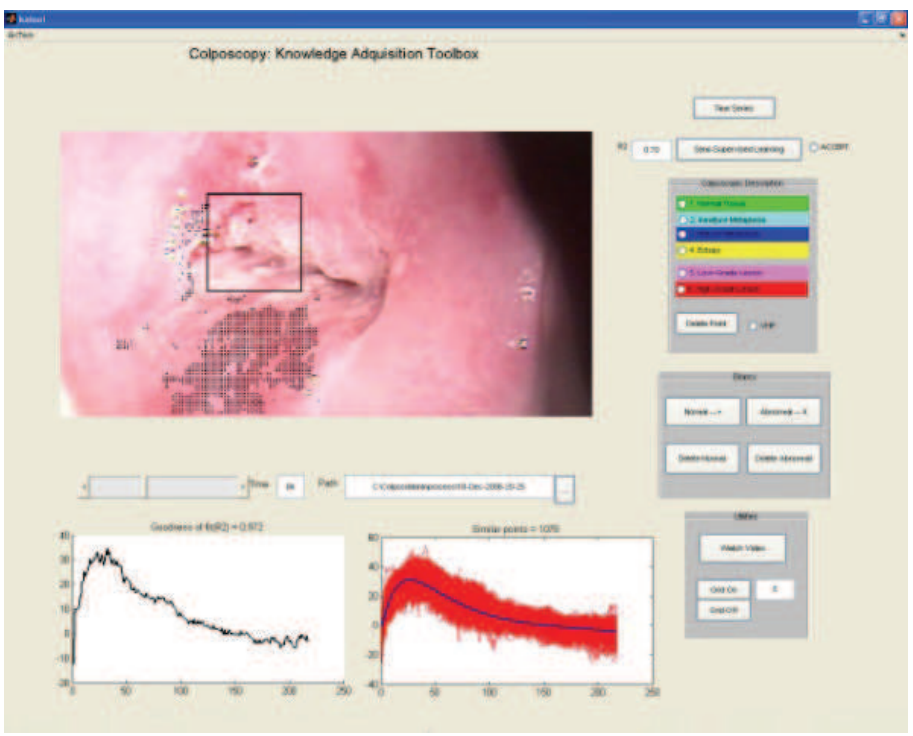

Fig. 3. Knowledge acquisition graphical interface. Using this tool, the user is able to assign a label to each representative region on tissue. The Awrf of each selected pixel of the image was displayed in a separate window. In a supervised fashion, for each selected pixel, a set of pixels with similar Awrf were suggested to the user in order to simplify the class labeling process.

respectively), immature metaplasia $(7,156)$, mature metaplasia $(5,189)$ and ectopia $(2,91)$. On the positive class are considered: low grade lesion $(26,1164)$ and high grade lesion $(3,123)$.

Once representative examples of tissue were labeled on each patient, a spatio-temporal data base was constructed containing, the patient identification, the observation's spatial position on the image, the class label and the temporal pattern extracted from the stereoscopic sequence relative to that position. The classification process was conducted in the WEKA software, in the Explorer module using the Naïve Bayes classifier. The classification performance was measured using leave-one-out cross-validation as explained above. Following the advice of the expert coposcopist, the class labels were binarized as follows: normal (normal tissue, immature metaplasia, mature metaplasia, ectopy), abnormal (low grade lesion, high grade lesion). Since the database contains more observations for the abnormal label, the occurrences were balanced using under sampling on the majority class (Pang-Ning et al., 2006; Han \& Kamber, 2001). The classification results are shown in table 2.

\begin{tabular}{|c|c|c|c|}
\hline Technique & Sensibility & Specificity & Accuracy \\
\hline NB (PLA) & 67 & 85 & 76 \\
\hline NB (Slopes) & 61 & 80 & 70 \\
\hline k-NN & 71 & 59 & 67 \\
\hline Colposcopists & 98 & 48 & 73 \\
\hline
\end{tabular}

Table 2. Classification performance. Performance values were computed as the mean values obtained on the classification over the 38 cases. 
The values shown for k-NN were obtained from (Acosta-Mesa et al., 2008) and are presented for comparative purposes.

Supervised learning was also developed over the entire image, that is to say, not only on those positions (pixels) selected by the expert during class labeling. In this analysis the classification was developed in a similar way as before using leave-one-out. However, in this case all the temporal patterns belonging to the entire image were including in the validation set. As is obvious, under this approach it is not possible to validate performance on the classification process because the entire image was not labeled in the class labeling task. However, it was visually evaluated by the expert, concluding that segmentation results are coherent with those introduced in the training set. To the best of our knowledge, only one work has reported the segmentation of the entire image using the aceto-white effect, however, they did not use the complete aceto-white temporal pattern (Young, 2008). As an example, the image segmentation displayed by the graphical interface developed by us is shown in figure 4 .

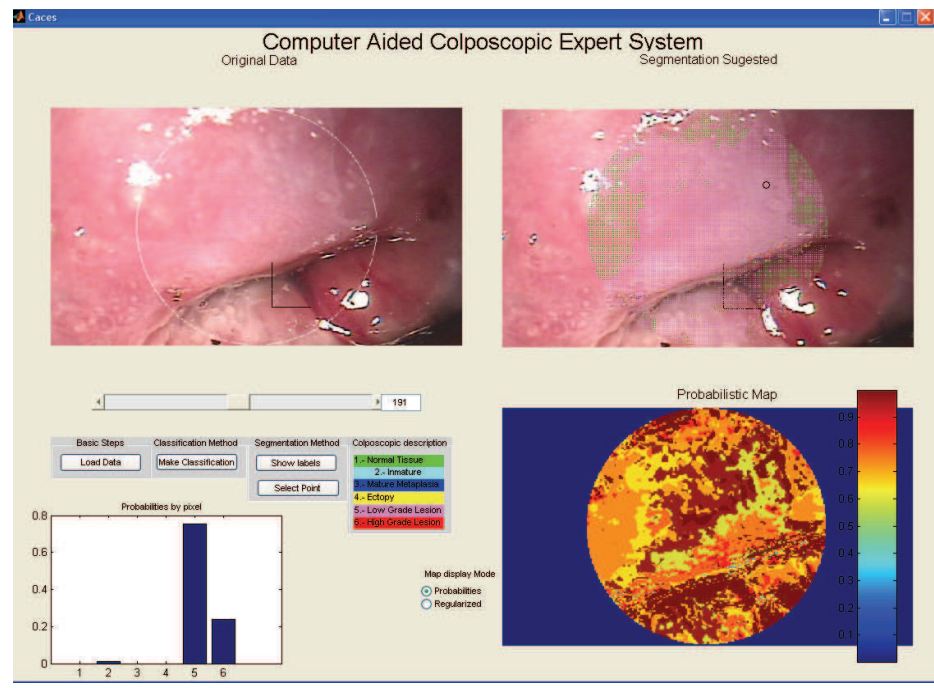

Fig. 4. Colposcopic image segmentation. The image at the left shows the original unknown colposcopic sequence given to the classifier. At the right, the image shows the labels suggested by the segmentation tool. Only the central part of the image, demarked by the circle, is analyzed to avoid artifacts introduced by illumination heterogeneities on the periphery. In the bottom left a histogram with the probability of a temporal pattern belonging to a tissue type (class) is shown. In the bottom right a geometric distribution of the likelihood of temporal patterns by type of tissue in them is also shown.

\section{Conclusions and future work}

In this pilot study our results show that normal and abnormal colposcopic findings can be discriminated using the temporal information intrinsic to the change of color occurred during aceto-whitening. The shape of the temporal patterns (Awrfs) can be used to segment a colposcopic image. Although some approaches have been proposed to analyze colposcopic images using temporal patterns, as far as we known, none of them has established a 
complete methodology to acquire and analyze colposcopic sequences in order to automatically segment the entire image. Our approach is one step toward it. However, more research in this field is needed in order to improve classification performance.

In this work, we present a way to automatically discriminate between normal and abnormal cervical tissue, using the machine learning supervised classification algorithm Naïve Bayes over the entire length of the aceto-white temporal pattern. Although this is one of the easiest machine learning classification techniques, it surpasses classification specificity reached by expert colposcopists, although the sensitivity reached by Naïve Bayes is lower than that reached by colposcopists. This behavior is presented in both the PLA method as PSA. These results can be explained because in the experiments discretization parameters were given in an arbitrary manner and this can affect the performance of classifiers.

As a continuation of this work we are using Time Series Data Mining (TSDM) techniques to explore compressed representations to facilitates classification (Last, Kandel, and Bunke 2004). Specifically, working with discrete representations of time series that can be treated using other approaches such as ID3, or its successor C4.5 algorithm, whereas the latter supports continuous values. Using these methods we want to explore the temporal pattern's feature that best separates the examples with the goal of achieving a better performance in the assessment of accuracy. We are also working on developing automatic mechanisms to determine the best scheme of discretization parameters. Finally, we continue growing the number of patients to be included in the training set in order to increase the space of cases.

\section{Acknowledgement}

The first author thanks the Mexican National Council for Science and Technology for the economic support of this project under the research grant: Fondo Sectorial de Investigación en Salud y Seguridad Social SSA/IMSS/ISSSTE-CONACYT (Salud-2003-C01-06).

\section{References}

Acosta-Mesa Héctor-Gabriel., Zitová Barbara., Ríos-Figueroa Homero., Cruz-Ramírez Nicandro., Marín-Hernández Antonio., Hernández-Jiménez Rodolfo., CocotleRonzón Bertha-Elvia., and Hernández Galicia Efraín. (2005). Cervical Cancer Detection Using colposcopic Images: a Temporal Approach. Proceeding of the Sixth International Conference on Computer Science (IEEE Computer Society Press). 158-164.

Acosta-Mesa Héctor-Gabriel, Cruz-Ramírez Nicandro, Hernández-Jiménez Rodolfo, GarcíaLópez Daniel-Alejandro. (2007). Modeling Aceto-White Temporal Patterns to Segment Colposcopic Images. Advances in Artificial Intelligence (LNCS. SpringerVerlag) 4478:548-555.

Acosta-Mesa Héctor-Gabriel, Cruz-Ramírez Nicandro, Hernández-Jiménez Rodolfo, AcetoWhite Temporal Pattern Classification using k-NN to Identify Precancerous Cervical Lesion in Colposcopic Images. Computers in Biology and Medicine. Elsevier, 2009. 39: p. 778-784.

Acosta-Mesa Héctor-Gabriel, Cruz-Ramírez Nicandro, Hernández-Jiménez Rodolfo, Cocotle-Ronzón Bertha-Elvia. (2008). Aceto-White Temporal Patterns Classification using k-NN to Identify Precancerous Cervical Lesion in Colposcopic Images. Submitted for publication.

Allgower Eugen L. and Georg Kurt. (1988). Estimates for Piecewise Linear Approximations of Implicity Defined Manifolds. SIAM Journal of Numerical Analysis. 24:452-469 
Anderson M., Jordan A. M, Sharp F. (1993). A Text and Atlas of Integrated Colposcopy. Mosby.

Balas, Costas. (2001). A Novel Optical Imaging Method for the Early Detection, Quantitative Grading, and Mapping of Cancerous and Precancerous Lesions of Cervix. IEEE Transactions in Biomedical Engineering. 48 (1):96-104.

Brown, Lisa Gottesfeld. (1992). A survey of Image Registration Techniques. ACM Computing Surveys (CSUR) 24 (4):325-376.

Burghardt Erich, Pickel Hellmuth, Girardi Frank. (2004). Primary care Colposcopy. Textbook and Atlas. New Year: Thieme Medical Publisher Incorporated.

Duda, R.O., Hart, Peter E., Stork, David G. (2001). Pattern Classification. John Wiley \& Sons, INC.

Friedman, N., D. Geiger, and M. Goldszmidt.(1997). Bayesian Network Classifiers. Machine Learning. 29: p. 131-163.

Han, J. and Kamber, M. (2001). Data Mining: Concepts and Techniques. Morgan Kaufmann Publishers.

Hastie Trevor, Tibshirani Robert, Friedman Jerome. (2001). The Elements of Statistical Learning (Data mining, Inference and Prediction), Springer series in statistics: Springer.

Keogh, E. and Pazzani, M. (2000). A simple dimensionality reduction technique for fast similarity search in large time series databases. In Proceedings of Pacific- Asia Conf. on Knowledge Discovery and Data Mining, pp 122-133

Last Mark, Kandel Abraham, and Horst Bunke. (2004). Data Mining in Time Series Databases. World Scientific.

Mitchell, Tom M. (1997). Machine Learning. Edited by McGraw-Hill.

Pogue Brian, Zelenchuk Alex, Harper Gregory, and Burke Eschel. (2001). Analysis of Acetic Acid-induced Whitening of High-grade Squamous Intraepitelial Lesions. Journal of Biomedical Optics. 6 (4):397-403.

Schmid-Saugeon Phillipe, Kaufman Howard B. , Zelenchuk Alex , Harper Dian M. 2004. Time-Resolved Imaging of Cervical Acetowhitening. Draft paper (Available in Electronic Form on: http://www.schmid-saugeon.com/publications/ Philippe\%20Schmid-Saugeon \%202003\%20Digital\%20colposcope.pdf )

Stefanaki Irene., Androniki. Tosca, George. Themelis, and Costas. Balas. 2001. In Vivo Detection of Human Papilloma Virus-Induced Lesions of Anogenital Area after Application of Acetic Acid: a Novel and Accurate Approach to a Trivial Method. Journal of Photochemistry and Photobiology. 65:115-121.

Tan Pang-Ning, Steinbach Michael, and Kumar Vipin. (2006). Introduction to Data Mining. Edited by A.-W. P. Co.

Tapia Antonio, Garrido Francisco, Gómez Octavio. (2006). Salud:México 2001-2005. Información estratégica por entidad federativa, edited by S. d. I. y. C. Dirección General de Evaluación del Desempeño: Secretaría de Salud.

Vlastos Anne-Thérèse, Richards-Kortum Rebecca, Zuluaga Andres and Follen Michele. (2002). New approaches to cervivcal cancer screening. Contemporary Ob/Gyn:87-103.

World Health Organization (WHO). (2007). Control integral del cáncer cervicouterino. Guía de prácticas esenciales. 1st ed. Suiza:WHO.

Young Sun Park, Follen Michele, Rhodes Andrea Milbourne Helen. (2008). Automated image analysis of digital colposcopy for the detection of cervical neoplasia. Journal of Biomedical Optics. 13(1) (January/February 2008):014029-1 / 014029-10.

Zitova Barbara, F.J. (2003). Image Registration Methods: a Survey. Image and vision computing 21 (11):977-1000. 


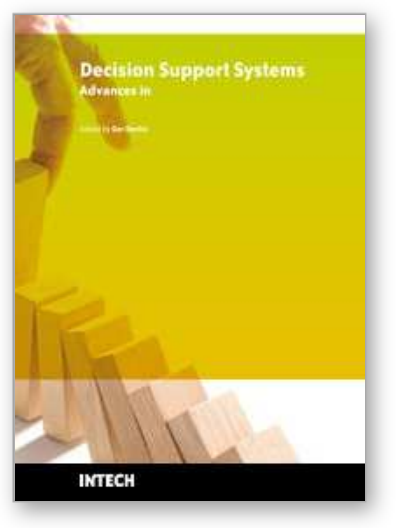

\author{
Decision Support Systems Advances in \\ Edited by Ger Devlin
}

ISBN 978-953-307-069-8

Hard cover, 342 pages

Publisher InTech

Published online 01, March, 2010

Published in print edition March, 2010

This book by In-Tech publishing helps the reader understand the power of informed decision making by covering a broad range of DSS (Decision Support Systems) applications in the fields of medical, environmental, transport and business. The expertise of the chapter writers spans an equally extensive spectrum of researchers from around the globe including universities in Canada, Mexico, Brazil and the United States, to institutes and universities in Italy, Germany, Poland, France, United Kingdom, Romania, Turkey and Ireland to as far east as Malaysia and Singapore and as far north as Finland. Decision Support Systems are not a new technology but they have evolved and developed with the ever demanding necessity to analyse a large number of options for decision makers (DM) for specific situations, where there is an increasing level of uncertainty about the problem at hand and where there is a high impact relative to the correct decisions to be made. DSS's offer decision makers a more stable solution to solving the semi-structured and unstructured problem. This is exactly what the reader will see in this book.

\title{
How to reference
}

In order to correctly reference this scholarly work, feel free to copy and paste the following:

Héctor-Gabriel Acosta-Mesa, Nicandro Cruz-Ramirez, Karina Gutierrez-Fragoso, Rocio-Erandi BarrientosMartinez and Rodolfo Hernandez-Jimenez (2010). Assessing the Possibility of Identifying Precancerous Cervical Lesions Using Aceto-White Temporal Patterns, Decision Support Systems Advances in, Ger Devlin (Ed.), ISBN: 978-953-307-069-8, InTech, Available from: http://www.intechopen.com/books/decision-supportsystems-advances-in/assessing-the-possibility-of-identifying-precancerous-cervical-lesions-using-aceto-whitetemporal-pa

\section{INTECH}

open science | open minds

\section{InTech Europe}

University Campus STeP Ri

Slavka Krautzeka 83/A

51000 Rijeka, Croatia

Phone: +385 (51) 770447

Fax: +385 (51) 686166

www.intechopen.com

\section{InTech China}

Unit 405, Office Block, Hotel Equatorial Shanghai

No.65, Yan An Road (West), Shanghai, 200040, China

中国上海市延安西路65号上海国际贵都大饭店办公楼405单元

Phone: +86-21-62489820

Fax: +86-21-62489821 
(C) 2010 The Author(s). Licensee IntechOpen. This chapter is distributed under the terms of the Creative Commons Attribution-NonCommercialShareAlike-3.0 License, which permits use, distribution and reproduction for non-commercial purposes, provided the original is properly cited and derivative works building on this content are distributed under the same license. 